\title{
The G292.0+1.8 pulsar wind nebula in the mid-infrared
}

\author{
D. A. Zyuzin ${ }^{1,2}$, A. A. Danilenko ${ }^{1}$, S. V. Zharikov ${ }^{3}$, and Yu. A. Shibanov ${ }^{1}$
}

\author{
1 Ioffe Physical Technical Institute, Politekhnicheskaya 26, St. Petersburg, 194021, Russia \\ e-mail: zyuzia@mail.ru \\ 2 Academical Physical Techonological University, Khlopina 2-8, St. Petersburg, 194021, Russia \\ 3 Observatorio Astronómico Nacional SPM, Instituto de Astronomía, UNAM, Ensenada, BC, Mexico
}

Received 21 August 2009 / Accepted 21 September 2009

\section{ABSTRACT}

\begin{abstract}
Context. G292.0+1.8 is a Cas A-like supernova remnant that contains the young pulsar PSR J1124-5916 powering a compact toruslike pulsar wind nebula visible in X-rays. A likely counterpart to the nebula has been detected in the optical VRI bands.

Aims. To confirm the counterpart candidate nature, we examined archival mid-infrared data obtained with the Spitzer Space Telescope. Methods. Broad-band images taken at 4.5, 8, 24, and $70 \mu \mathrm{m}$ were analyzed and compared with available optical and X-ray data.

Results. The extended counterpart candidate is firmly detected in the 4.5 and $8 \mu \mathrm{m}$ bands. It is brighter and more extended in the bands than in the optical, and its position and morphology agree well with the coordinates and morphology of the torus-like pulsar wind nebula in X-rays. The source is not visible in 24 and $70 \mu \mathrm{m}$ images, which are dominated by bright emission from the remnant shell and filaments. We compiled the infrared fluxes of the nebula, which probably contains a contribution from an unresolved pulsar in its center, with the optical and X-ray data. The resulting unabsorbed multiwavelength spectrum is described by power laws of significantly steeper slope in the infrared-optical than in X-rays, implying a double-knee spectral break between the optical and $\mathrm{X}$-rays. The 24 and $70 \mu \mathrm{m}$ flux upper limits suggest a second break and a flatter spectrum at the long wavelength limit. These features are common to two other pulsar wind nebulae associated with the remnants B0540-69.3 and 3C 58 and observed in all three ranges. Conclusions. The position, morphology, and spectral properties of the detected source allow us to confirm that it is the infrared-optical counterpart to both the pulsar and its wind nebula system in the G292.0+1.8 supernova remnant.
\end{abstract}

Key words. ISM: supernova remnants - pulsars: individual: PSR J1124-5916 - stars: winds, outflows - infrared: general radiation mechanisms: non-thermal - ISM: individual objects: G292.0+1.8

\section{Introduction}

Infrared (IR) observations can provide valuable information about the poorly understood physical processes responsible for multiwavelength radiation from rotation-powered neutron stars and pulsar wind nebulae (PWNe). However, until now only a few of these objects have been detected in the IR. The most well studied Crab-pulsar and its torus-like PWN exhibit almost a flat spectrum in the IR (Temim et al. 2006, 2009), while some bright structures of the PWN, such as a knot south of the pulsar, exhibit a steep flux increase towards longer wavelengths (Sollerman 2003; Sandberg \& Sollerman 2009). In contrast to the emission of the Crab-pulsar itself, the emission from three older pulsars detected in the near-IR (Vela, B0656+14, Geminga) show a flux increase with wavelength (Shibanov et al. 2003, 2006).

Two young Crab-like pulsar+PWN systems associated with supernova remnants (SNRs) B0540-69.3 and 3C 58, observed in the mid-IR with the Spitzer telescope, exhibited an even steeper flux increase towards the IR (Williams et al. 2008; Slane et al. 2008; Shibanov et al. 2008). Their optical and IR data can be described by a single power law, whose slope and intensity differ significantly from those observed in X-rays suggesting a double-knee break in the multiwavelength spectra of both systems between the optical and X-rays (Serafimovich et al. 2004; Shibanov et al. 2008). The emission from the Crab contains only one break within the same range. More complex energy distributions of radiating relativistic particles in the two former systems may be resposible for this difference. IR observations of other pulsar-PWN systems can help us to understand whether the double-knee break is a more common feature of these systems than the single break seen in the Crab.

The young PSR J1124-5916 was only recently discovered in the radio (Camilo et al. 2002) and X-rays (Hughes et al. 2001, 2003). It is associated with SNR G292.0+1.8 (MSH 1154), which is the third oxygen-rich SNR to be detected in the Galaxy after Cas A and Puppis A. In a similar way to the Crabpulsar, PSR J1124-5916 powers a torus-like X-ray PWN with a jet (Hughes et al. 2001; Safi-Harb \& Gonzalez 2002; Hughes et al. 2003; Park et al. 2007). The characteristic age of the pulsar, $\tau \approx 2900 \mathrm{yr}$, which is consistent with $2700-3700 \mathrm{yr}$ age of the SNR (Camilo et al. 2002; Chevalier 2005), and its spindown luminosity, $\dot{E} \approx 1.2 \times 10^{37} \mathrm{ergs} \mathrm{s}^{-1}$, rank this pulsar as the sixth youngest and the eighth most energetic among the all rotation-powered pulsars known. Deep optical images obtained last year with the VLT detected a likely candidate to the optical counterpart of the pulsar+PWN system in VRI bands (Zharikov et al. 2008). The major axis of an elliptically shaped counterpart candidate coincides with the major axis of the torus-like PWN, which is seen in X-rays almost edge-on. Smaller source extents suggest that only the brightest inner part of the X-ray PWN containing the pulsar was detected in the optical range.

Using Spitzer archival images of the G292.0+1.8 field, we report the detection of the same source in the mid-IR. Similar source morphology to that in X-rays and a single power law spectral energy distribution in the optical and IR show that 
it is indeed the optical-mid-IR counterpart of the G292.0+1.8 pulsar+PWN system. The IR observations are described in Sect. 2, the results are presented and discussed in Sects. 3 and 4.

\section{Observations}

G292.0+1.8 was imaged with the Spitzer telescope at several observing sets from March 13 to April 152008 using the Infrared Array Camera (IRAC) and Multiband Imaging Photometer for Spitzer (MIPS) ${ }^{1}$. The data $^{2}$ were retrieved from the Spitzer archive. Various parts of the SNR were detected in all four IRAC channels, although its central part, containing the pulsar, was imaged in only the second and fourth bands with effective wavelengths of $4.5 \mu \mathrm{m}$ and $8.0 \mu \mathrm{m}$, respectively. We used the IRAC post-BCD calibrated mosaic images with an effective image scale of 1'.2 per pixel and field of view (FOV) of 7'.5 $\times 7$ '.5. Two separate sets of observations were obtained with the MIPS at the effective wavelengths of $24 \mu \mathrm{m}$ and $70 \mu \mathrm{m}$ to image the entire SNR. We regenerated the MIPS post-BCD calibrated mosaic images from respective $\mathrm{BCD}$ data using the $\mathrm{MOPEX}^{3}$ tool. The resulting image scales are 2.' 4 per pixel for the first, and $4^{\prime \prime} 0$ for the second band with the FOV of 13 ' $3 \times 12$ '. 8 for both bands. The effective exposures were $\approx 3500$ s per pixel for both IRAC bands, $\approx 500 \mathrm{~s}$ for $24 \mu \mathrm{m}$, and $\approx 380 \mathrm{~s}$ for $70 \mu \mathrm{m}$ MIPS bands.

\section{Results}

\subsection{Identification of the pulsar / PWN counterpart candidate}

As a first step, to check the Spitzer pointing accuracy, we applied astrometric referencing to the IRAC images. In the IRAC FOV, eight unsaturated isolated astrometric standards from the USNO-B1 catalog were selected as reference points. Their catalog and image positional uncertainties for both coordinates were $\lesssim 0$ '.2 and $\lesssim 0$ '.5, respectively. IRAF ccmap/cctran tasks were used to find plate solutions. Formal $r m s$ uncertainties of the astrometric fit were $\lesssim 0$, . 2 with maximal residuals of $\lesssim 00^{\prime} .5$ for both coordinates and both IRAC bands. After astrometric transformations the shifts between the original and transformed images were $\lesssim 0$. $^{\prime} 4$ (or less than 0.3 of the pixel scale), ensuring the almost perfect pointing accuracy of the Spitzer observations. Combining all uncertainties, a conservative estimate of $1 \sigma$ referencing uncertainty is $\lesssim 0^{\prime} .5$ (or less than 0.4 of the IRAC pixel scale) in both coordinates for both bands. This is about twice as poor as the astrometric uncertainties estimated by Zharikov et al. (2008) for the deepest available Chandra/ACIS-I X-ray $(\$ 0 . ' 27)$ and VLT/FORS2 $\left(\$ 0\right.$ '. $\left.^{\prime} 15\right)$ optical images of G292.0+1.8. Nevertheless, this is sufficient to identify positionally the objects in the mid-IR, optical, and X-rays on a subarcsecond accuracy level.

The region containing the pulsar is shown in Fig. 1, where we compare the IRAC mid-IR images with the X-ray and optical images obtained with Chandra/ACIS-I and VLT/FORS2 (Zharikov et al. 2008). In both IRAC bands at the pulsar position, marked by a cross, we detect a faint extended source. The FWHM of the IR point spread function varies from 2 .' 1 to 2 .'5, and the extended structure of the source, which subtends up to $\approx 10.5$, is clearly resolved. In the $8 \mu \mathrm{m}$ image, which is less contaminated by background stars, the object has

\footnotetext{
1 See http://ssc.spitzer.caltech.edu

2 PROGID 40583, PI Ghavamian.

${ }^{3}$ http://ssc.spitzer. caltech.edu/postbcd/mopex.html
}

an elliptical shape, as can also be seen from its contours overlaid on the X-ray and optical images ${ }^{4}$. The coordinates of the source center, derived from the source surface brightness fit in $8 \mu \mathrm{m}$ band by elliptical isophotes, are $\mathrm{RA} \approx 11: 24: 39.129$ and Dec $\approx-59: 16: 19.51$. Accounting for the astrometric uncertainties, this is in good agreement with the X-ray position of the pulsar, $\mathrm{RA} \approx 11: 24: 39.183$ and Dec $\approx-59: 16: 19.41$, and with the central position of the optical pulsar/PWN counterpart candidate, $\mathrm{RA} \approx 11: 24: 39.216$ and Dec $\approx-59: 16: 19.60$ (Zharikov et al. 2008). The source ellipticity and position angle are compatible with the structure of the brightest inner part of the X-ray PWN, which has been interpreted as a Crab-like PWN torus seen almost edge-on (Hughes et al. 2003).

The position and morphology of the detected mid-IR source allow us to consider whether it is a true counterpart to the pulsar+PWN system. A faint jet-like structure extended south of the torus and a bright SNR filament visible N-E of the PWN in $\mathrm{X}$-rays are not detected in the mid-IR. In the frames presented in Fig. 1, we note only one additional cross-identification, an object o1, showing a point-like spatial profile in X-rays but a nonstellar structure in the IR. This may be a background galaxy. Star-subtracted optical and mid-IR images (bottom row panels of Fig. 1) show that at the pulsar position we see the same extended elliptical object in both ranges. This confirms the reality of its optical detection in the region that is extremely crowded by background objects (Zharikov et al. 2008). Most nearby stars contaminating the source flux in the optical becomes fainter in the IR, while the brightness of the source itself does not decrease, enabling its detection in the mid-IR at a higher significance level. As in the optical, its surface brightness distribution reaches a peak at the pulsar position. We cannot resolve any point-like object there, but the peak is probably associated with the contribution from the pulsar. Some blurring is caused by a lower mid-IR spatial resolution compared to the optical one. However, the object sizes along its major axis corresponding to $\gtrsim 90 \%$ of the enclosed flux are significantly larger in the IR, $\approx 10$.' 5 , than in the optical, $\approx 3^{\prime \prime}$. This suggests that the source becomes brighter with wavelength (see below Sects. 3.2 and 3.3), revealing its fainter outer regions. At $8 \mu \mathrm{m}$, it becomes comparable in size and shape to the torus part of the PWN seen in X-rays.

Our counterpart candidate is not detected in the MIPS bands, where the emission from filaments and the outer shell of the SNR strongly dominates over other sources in contrast to the shorter wavelength IRAC bands. Some faint structure may be present at the pulsar position, particularly in the $24 \mu \mathrm{m}$ image. However, the poorer spatial resolution of MIPS does not allow us to determine, whether it is related to the candidate or to a faint peninsulalike part of a bright nearby filament of the remnant.

\subsection{Photometry}

Elliptical aperture photometry of the detected source was performed on the star-subtracted IRAC images using IRAF polyphot tasks in accordance with prescriptions and zeropoints given in the IRAC Observers Manual ${ }^{5}$. The elliptical apertures were obtained from the source surface brightness fit at $8 \mu \mathrm{m}$ by elliptical isophotes using IRAF isophot tasks. The ellipticities

\footnotetext{
${ }^{4}$ In both images, the contours are identical, but the internal IR contour seen in the optical falls inside the X-ray PWN and is not visible on chosen scale levels in the X-ray image.

5 See, e.g., http://ssc. spitzer. caltech. edu/archanaly/quickphot. html
} 

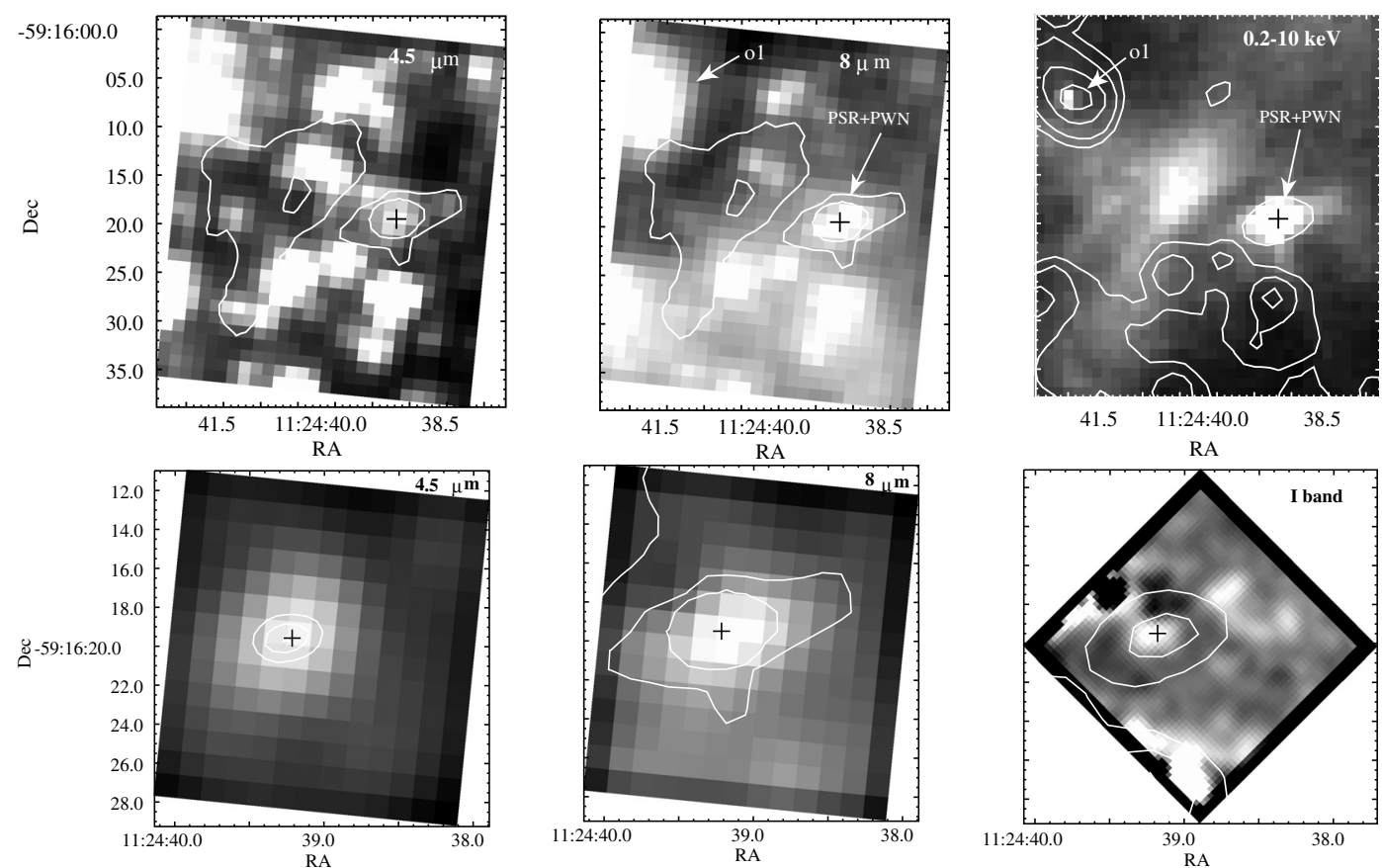

Fig. 1. Top row: central $\sim 35^{\prime \prime} \times 40^{\prime \prime}$ fragment of G292.0+1.8, containing PSR J1124-5916 and its PWN as seen with the Spitzer/IRAC and Chandra/ACIS-I at $4.5 \mu \mathrm{m}$ and $8 \mu \mathrm{m}$, and in $0.2-10 \mathrm{keV} \mathrm{X-ray} \mathrm{range,} \mathrm{respectively.} \mathrm{X-ray} \mathrm{position} \mathrm{of} \mathrm{the} \mathrm{pulsar} \mathrm{is} \mathrm{marked} \mathrm{by} \mathrm{the} \mathrm{cross.} \mathrm{Contours}$ from the X-ray image are overlaid on the mid-IR images, while in X-rays the contours are from the $8 \mu \mathrm{m}$ image. Arrows indicate the pulsar+PWN system and a background object o1 in X-rays and their mid-IR counterparts. Bottom row: zoomed fragments of the same mid-IR images showing a $\sim 17^{\prime \prime} \times 17^{\prime \prime}$ region around the pulsar and respective $I$ band optical image obtained with the VLT/FORS2. Background stars were subtracted. Contours of the optical pulsar+PWN counterpart candidate are overlaid on $4.5 \mu \mathrm{m}$ image. Contours on the $I$ image are from $8 \mu \mathrm{m}$, while on the $8 \mu \mathrm{m}$ image the X-ray contours are overlaid. The images are smoothed with a Gaussian kernel of two-three pixels. The coordinates and morphology of the source seen at the pulsar position in the optical and mid-IR suggest that it is the counterpart of the X-ray pulsar+PWN system.

varied with the ellipse sizes in a range of $0.4-0.5$ and the semimajor axis of the outer isophote was found to be $\$ 6$ pixels (7'.2). The annulus for backgrounds was 8-23 pixels centered on the pulsar position. A typical semi-major radius, where the curves of growth saturate, was about 5 pixels for both IRAC bands. We reiterated the star subtraction process, varied the background region, and used circular apertures. The differences in the magnitudes obtained were similar to the measurement statistical errors and they were included in the resulting uncertainties. A few percent of the extended source aperture corrections were insignificant compared to the resulting $\approx 20-30 \%$ flux error budget.

The $3 \sigma$ upper limits to the source magnitudes in the MIPS bands were estimated using the standard deviations and inherent flux variations in the possible faint part of the remnant filament within the same elliptical apertures as in the IRAC bands. Both ways provided similar values. For $70 \mu \mathrm{m}$, we used a postBCD filtered calibrated mosaic image generated by us with the MOPEX tool. The magnitudes were converted into fluxes in physical units and the results are summarized in Table 1. Our $70 \mu \mathrm{m}$ limit is slightly above the $5 \sigma$ flux confusion limit of $1500 \mu \mathrm{J}$ estimated for this MIPS band by Frayer et al. (2006).

\subsection{Multiwavelength spectrum}

Using the mid-IR, optical, and X-ray data, we compiled a tentative multiwavelength spectrum of the pulsar/PWN system. We dereddened the observed optical/IR fluxes using a most plausible interstellar extinction range $1.86 \lessgtr A_{V} \lesssim 2.10$ discussed in detail by Zharikov et al. (2008). Standard extinction curve (Cardelli et al. 1989) and average $A_{\lambda} / A_{K}$ ratios provided especially for the
Table 1. Observed magnitudes and fluxes for the presumed infrared/optical pulsar/PWN counterpart of J1124-5916, and de-reddened fluxes for the $A_{V}$ range of 1.86-2.10.

\begin{tabular}{|c|c|c|c|}
\hline $\begin{array}{l}\lambda_{\text {eff }}(\text { band }) \\
(\mu \mathrm{m})\end{array}$ & $\begin{array}{l}\text { Mag. } \\
\text { observed }^{a} \\
\text { (mag) }^{\text {mag }}\end{array}$ & $\begin{array}{l}\text { log Flux } \\
\text { observed }^{a} \\
(\mu \mathrm{J})\end{array}$ & $\begin{array}{l}\text { log Flux } \\
\text { de-reddened }{ }^{a} \\
(\mu \mathrm{J})\end{array}$ \\
\hline & Infrared & Spitzer & \\
\hline 70 & $\gtrsim 6.8$ & $\$ 3.2$ & $\$ 3.2$ \\
\hline 24 & $\gtrsim 10.2$ & $\$ 2.8$ & $\lesssim 2.85$ \\
\hline 8.0 & $14.2(3)$ & $2.13(16)$ & $2.18\left({ }_{-17}^{+16}\right)$ \\
\hline 4.5 & $15.9(4)$ & $1.90(21)$ & $1.95\left({ }_{-22}^{+21}\right)$ \\
\hline $0.77(I)$ & $\begin{array}{l}\text { Optical } \\
23.12(13)\end{array}$ & $\begin{array}{l}\text { VLT } \\
0.13(5)\end{array}$ & $0.66\left({ }_{-10}^{+6}\right)$ \\
\hline $0.66(R)$ & $24.12(13)$ & $-0.17(5)$ & $0.51\left(_{-12}^{+7}\right)$ \\
\hline $0.55(V)$ & $24.29(13)$ & $-0.16(5)$ & $0.66\left(\left(_{-13}^{+7}\right)\right.$ \\
\hline
\end{tabular}

${ }^{a}$ Numbers in brackets are $1 \sigma$ uncertainties referring to last significant digits quoted.

IRAC bands (Indebetouw et al. 2005; Fraherty et al. 2007) were applied. The results are presented in Table 1, where we also include the optical data obtained by Zharikov et al. (2008).

To compare the fluxes in different ranges from the same physical region, we extracted the pulsar/PWN X-ray spectrum from the archival Chandra/ACIS-I data ${ }^{6}$ using the same elliptical aperture, as for the mid-IR photometry of the detected source, with the semimajor axis of $5^{\prime \prime} .2$, ellipticity $\approx 0.5$, and the $\mathrm{PA} \approx-70^{\circ}$. An absorbed power law spectral model

${ }_{6}^{6}$ Obs 6677-6680, 8221, 8447, 530 ks exposure, PI S. Park 


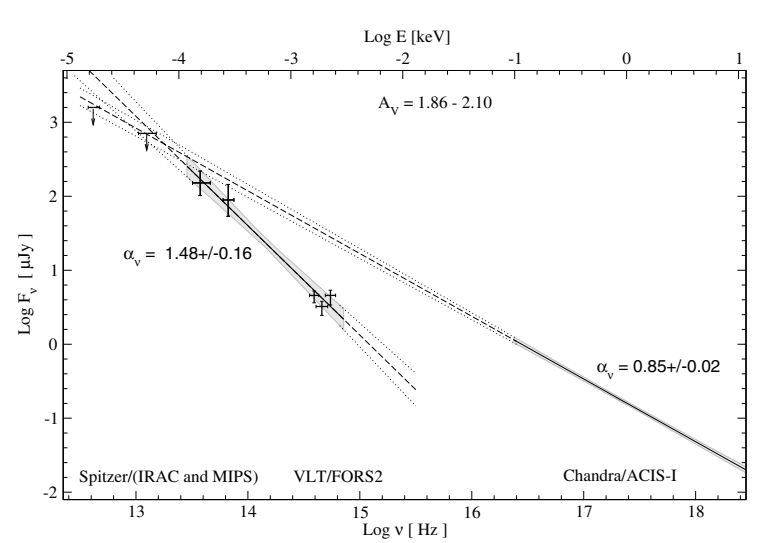

Fig. 2. Tentative unabsorbed multiwavelength spectrum for the inner part of the torus region of the G292.0+1.8 pulsar/PWN system compiled from the data obtained with different instruments, as indicated in the plot. Errorbars of the dereddened optical and mid-IR fluxes include the $A_{V}$ uncertainty range shown at the top. The VLT and IRAC data are fitted by a single powerlaw, whose spectral index $\alpha_{v}$ (defined by $\left.F_{v} \propto v^{-\alpha_{\nu}}\right)$ is significantly greater than that for X-rays, suggesting a double knee spectral break between the optical and X-rays. The MIPS flux upper limits are below the low frequency extension of this fit and imply a second break at $\lambda \approx 20 \mu \mathrm{m}$. Solid lines show the best spectral fits, while grey polygons, and dashed and dotted lines are the fit uncertainties and their extensions outside the frequency ranges involved in the fits.

provided a statistically acceptable fit with a photon spectral index $\Gamma=1.85 \pm 0.02$, absorbing column density $N_{\mathrm{H}}=(3.4 \pm$ $0.1) \times 10^{21} \mathrm{~cm}^{-2}$, and normalization constant $C=(2.4 \pm 0.1) \times$ $10^{-4}$ photons $\mathrm{cm}^{-2} \mathrm{~s}^{-1} \mathrm{keV}^{-1}$. The fit had $\chi^{2}=1.1$ per degree of freedom (d.o.f.) and the unabsorbed integral flux was $1.0 \times 10^{-12} \mathrm{erg} \mathrm{cm}^{-2} \mathrm{~s}^{-1}$ in $0.3-10 \mathrm{keV}$ range. The resulting $\Gamma$ and $N_{H}$ are compatible with those of Zharikov et al. (2008), while the normalization constant and integral flux are about twice as high. This is because of a larger extraction aperture than in their work, where the aperture was chosen to fit the smaller source extent in the optical range. The optical fluxes are unchanged by the aperture increase since the likely counterpart is more compact in the optical than in the IR.

The resulting unabsorbed spectrum is presented in Fig. 2. The counterpart candidate spectral energy distribution (SED) in the IR-optical range shows a steep flux increase towards the midIR. The fluxes in this range can be fitted by a single power-law with a spectral index $\alpha_{v} \approx 1.5\left(\chi^{2} \approx 0.5\right.$ per d.o.f. $)$, implying a nonthermal nature of the emission. This is expected for PWNe, where synchrotron radiation from relativistic particles accelerated at the pulsar wind termination shock is considered as the main radiative process responsible for the continuum emission of the nebulae. Accounting for possible contamination of the $V$ flux by O III emission from the SNR (Zharikov et al. 2008), we excluded this band from the fit. This leads to only a marginal improvement of the fit with insignificant steepening of the spectral slope within the uncertainties shown in the plot. The observed SED cannot be produced by overlapping field stars of the same brightness. Our test measurements of nearby faint stars show that in the latter case the spectral slope would be a positive, flat, or curved.

As seen from Fig. 2, the optical and mid-IR fluxes of the suggested counterpart are below the low frequency extrapolation of the pulsar/PWN spectrum in X-rays. This implies a doubleknee spectral break between the optical and X-rays. At the same time, the flux upper limits in the MIPS bands are below the low frequency extrapolation of the IRAC-optical SED, suggesting a second break near $20 \mu \mathrm{m}$ after which the spectrum become flatter or goes down with the frequency decrease. These changes in the spectral index and breaks are similar to those observed for PWNe around young pulsars in SNRs 3C 58 and B054069.3 (Serafimovich et al. 2004; Shibanov et al. 2008; Slane et al. 2008; Williams et al. 2008). Together with the source morphology, this is a strong evidence that the detected source is the true mid-IR and optical counterpart of the pulsar/PWN system in the G292.0+1.8 supernova remnant.

\section{Discussion}

These broad-band Spitzer observations have allowed us to confirm the pulsar/PWN counterpart nature of a faint optical nebulosity detected early at the PSR J1124-5916 position with the VLT (Zharikov et al. 2008). Its similar source morphology in the mid-IR, optical, and X-rays, as well as its spectral properties make any alternative interpretation discussed in Zharikov et al. (2008) (SNR filament, faint background spiral galaxy) very unlikely. The mid-IR data have enabled us to establish the long wavelength SED of the nebula, which was very uncertain based only on the optical data, and conclude that the multiwavelength spectrum of the J1124-5916 pulsar/PWN system from the midIR to X-rays is similar to the spectra of pulsar/PWN systems in the SNRs B0540-69.3 and 3C 58. Forthcoming high spatial resolution near-IR observations of the pulsar field will allow us to decrease the rather large uncertainties of the IR-optical spectral slope and possible distinguish the pulsar from the PWN.

Our results increase the number of PWNe identified in the mid-IR from three to four, and show that these objects can be significantly brighter in the IR than in the optical, making the IR range promising for the study of the PWNe. The presence of the double-knee breaks in the spectra between the optical and $\mathrm{X}$-rays in the three of four torus-like PWNe makes this feature regular and notable. This is distinct from the Crab-PWN and has to be taken into account in the modeling of the PWNe when understanding their structure and emission nature.

Acknowledgements. We are grateful to anonymous referee for useful comments improving the paper. The work was partially supported by RFBR (grants 0802-00837a, 09-02-12080), NSh-2600.2008.2, CONACYT 48493 and PAPIIT IN101506 projects, and by the German $D F G$ project number Ts $17 / 2-1$. DAZ was supported by St Petersburg Goverment grant for young scientists (2.3/0405/008).

\section{References}

Camilo, F., Manchester, R. N., Gaensler, B. M., et al. 2002, ApJ, 567, L71 Cardelli, J. A., Clayton, G. C., \& Mathis, J. S. 1989, ApJ, 345, 245 Chevalier, R. 2005, ApJ, 619, 839

Flaherty, K. M., Pipher, J. L., Megeath, S. T., et al. 2007, ApJ, 663, 1069 Frayer, D. T., Huynh, M. T., \& Chary, R. 2006, ApJ, 647, L9 Hughes, J. P., Slane, P. O., Burrows, D. N., et al. 2001, ApJ, L153 Hughes, J. P., Slane, P. O., Park, S., et al. 2003, ApJ, 591, L139 Indebetouw, R., Mathis, J. J., Babler, B. L., et al. 2005, ApJ, 619, 931 Park, S., Hughes, J. P., Slane, P. O., et al. 2007, ApJ, 670, L121 Safi-Harb, S., \& Gonzalez, M. E. 2002, in X-rays at Sharp Focus: Chandra Science, Symp. ASP Conf. Ser., 262, ed. E. Schlegel, \& S. D. Vrtilek Sandberg, A., \& Sollerman, J. 2009, A\&A, 504, 525

Serafimovich, N., Shibanov, Yu. A., Lundqvist, P., et al. 2004, A\&A, 425, 1041 Shibanov, Yu. A., Koptsevich, A. B., Sollerman, J., et al. 2003, A\&A, 406, 645 Shibanov, Y. A., Zharikov, S. V., Komarova, V. N., et al. 2006, A\&A, 448, 313 Shibanov, Yu. A., Lundqvist, N., Lundqvist, P., et al. 2008, A\&A, 486, 273 Slane, P., Helfand, D. J., Reynolds, S. P., et al. 2008, ApJ, 676, L33 Sollerman, J. 2003, A\&A, 46, 639

Temim, T., Gehrz, R. D., Woodward, C. E., et al. 2006, AJ, 132, 1610 Temim, T., Gehrz, R. D., Woodward, C. E., et al. 2009, AJ, 137, 5155 Williams, B. J., Borkowski, K. J., Reynolds, S. P., et al. 2008, ApJ, 687, 1054 Zharikov, S., Shibanov, Yu., Zyuzin, D. A., et al. 2008, A\&A, 479, 793 